\title{
STRONG CONSISTENCY OF ESTIMATORS FOR HETEROSCEDASTIC PARTLY LINEAR REGRESSION MODEL UNDER DEPENDENT SAMPLES
}

\author{
HAN-YING LIANG \\ Tongji University \\ Department of Applied Mathematics \\ Shanghai 200092, PR of China \\ E-mail: hyliang83@hotmail.com \\ BING-YI JING \\ Hong Kong University of Science and Technology \\ Department of Mathematics \\ Clear Water Bay, Kowloon, Hong Kong \\ E-mail: majing@ust.hk
}

(Received July, 2001; Revised February, 2002)

\begin{abstract}
In this paper we are concerned with the heteroscedastic regression model $\left.y_{i}=x_{i} \beta+g\left(t_{i}\right)+\sigma_{i} e_{i} \quad 1 \leq i \leq n\right)$ under correlated errors $e_{i}$, where it is assumed that $\sigma_{i}^{2}=f\left(u_{i}\right)$, the design points $\left(x_{i}, t_{i}, u_{i}\right)$ are known and nonrandom, and $g$ and $f$ are unknown functions. The interest lies in the slope parameter $\beta$. Assuming the unobserved disturbance $e_{i}$ are negatively associated, we study the issue of strong consistency for two different slope estimators: the least squares estimator and the weighted least squares estimator.

Key words: Partial Linear Model, Negatively Associated Sample, Weighted Least Square Estimator, Strong Consistency.

AMS subject classifications: $62 \mathrm{G} 05$.
\end{abstract}

\section{Introduction}

Consider the following heteroscedastic partial linear regression model:

$$
y_{i}=x_{i} \beta+g\left(t_{i}\right)+\sigma_{i} e_{i}, \quad 1 \leq i \leq n,
$$

where $\beta$ is an unknown parameter of interest, $\sigma_{i}^{2}=f\left(u_{i}\right),\left(x_{i}, t_{i}, u_{i}\right)$ are nonrandom design points, $y_{i}$ are the response variables, $\epsilon_{i}$ are random errors, and $f(\cdot)$ and $g(\cdot)$ are unknown functions defined on a closed interval $I$ of the real line $R$.

Clearly, the model (1.1) reduces to the partial linear model when $\sigma_{i}^{2}=\sigma^{2}$ and $e_{i}$ are i.i.d. This was first introduced by Engle et al. [11], and further studied by Heckman [14], Speckman [25], Chen [7], Chen and Shiau [8, 9], Hong and Cheng [15, 16], Hamilton and Truong [13], and Mammen and Van de Geer [20] among others. Various estimators for $\beta$ and $g(\cdot)$ were given by using different methods such as the kernel method, the penalized spline method, the piecewise constant smooth method, the smoothing splines and the trigonometric series approach. 
If $g(t) \equiv 0, \sigma_{i}^{2}=f\left(x_{i}\right)$, and $e_{i}$ are i.i.d., then the model (1.1) boils down to the heteroscedastic linear model. Asymptotic properties for the weighted least squares estimate of $\beta$, based on kernel estimate and nearest neighbor estimate of $f(\cdot)$ were studied by Caroll [5], Robinson [22], and Carroll and Hardle [6].

For the more general case where we only assume that $e_{i}$ are i.i.d. in the model (1.1), asymptotic properties have also been studied by various authors. For instance, Chen, Ren, and $\mathrm{Hu}$ [10] and Gao, Chen and Zhao [12] established the strong consistency and the asymptotic normality, respectively, for the least squares estimator and weighted least squares estimator of $\beta$, based on nonparametric estimates of $f(\cdot)$ and $g(\cdot)$.

All the work discussed so far assumes that the errors $e_{i}$ are independent. The purpose of this paper is to study some asymptotic properties of the slope estimators when $e_{i}$ are correlated. In particular, we shall study the strong consistency of several commonly used estimators of the slope $\beta$ when the errors $e_{i}$ are negatively associated (NA), whose definition will be given later in the paper.

The paper is organized as follows. In Section 2, we shall introduce the least squares estimators (LSE) and weight least squares estimators (WLSE) of the slope parameter $\beta$. The main results are given in Section 3, while their proofs will be provided in Section 4.

\section{2. (Weighted) Least Squares Estimations}

Let $W_{n j}(t)(1 \leq j \leq n)$ be the weight functions. For instance, one could choose the weight function to be

$$
W_{n j}(t)=K\left(\frac{t-T_{j}}{h}\right) / \sum_{k=1}^{n} K\left(\frac{t-T_{k}}{h}\right),
$$

where $K(\cdot)$ is a known nonnegative function and $h \equiv h(n)>0$ is a sequence of bandwidths.

Define

$$
S_{n}^{2}=\sum_{i=1}^{n} \widetilde{x}_{i}^{2}, \widetilde{x}_{i}=x_{i}-\sum_{j=1}^{n} W_{n j}\left(t_{i}\right) x_{j}, \widetilde{y}_{i}=y_{i}-\sum_{j=1}^{n} W_{n j}\left(t_{i}\right) y_{j}
$$

Then the LSE of $\beta, \widehat{\beta}_{n}$, and the corresponding nonparametric regression estimator of $g$, $\widehat{g}_{n}(t)$, are given by

$$
\widehat{\beta}_{n}=\sum_{i=1}^{n} \widetilde{x}_{i} \widetilde{y}_{i} / S_{n}^{2}, \quad \widehat{g}_{n}(t)=\sum_{i=1}^{n} W_{n i}(t)\left(y_{i}-x_{i} \widehat{\beta}_{n}\right) .
$$

If we assume that $f(\cdot)$ is known, then the weighted LSE of $\beta, \widetilde{\beta}_{n}$ and the corresponding nonparametric regression estimator of $g, \widetilde{g}_{n}(t)$, are given by

$$
\widetilde{\beta}_{n}=\sum_{i=1}^{n} a_{i} \widetilde{x}_{i} \widetilde{y}_{i} / T_{n}^{2}, \quad \widetilde{g}_{n}(t)=\sum_{i=1}^{n} W_{n i}(t)\left(Y_{i}-x_{i} \widetilde{\beta}_{n}\right),
$$

where $T_{n}^{2}=\sum_{i=1}^{n} a_{i} \widetilde{x}_{i}^{2}$, and $a_{i}=1 / f\left(u_{i}\right), 1 \leq i \leq n$.

If $f(\cdot)$ is unknown, then its nonparametric estimate can be given by 


$$
\widehat{f}_{n}(u)=\sum_{i=1}^{n} \widehat{W}_{n i}(u)\left(\widetilde{y}_{i}-\widetilde{x}_{i} \widehat{\beta}_{n}\right)^{2},
$$

where $\widehat{W}_{n i}(u)$ are weight functions. Assume $\min _{1 \leq i n}\left|\widehat{f}_{n}\left(u_{i}\right)\right|>0$, then the weighted LSE of $\beta, \bar{\beta}_{n}$, and the corresponding nonparametric regression estimator of $g, \bar{g}_{n}(t)$, are given by

$$
\bar{\beta}_{n}=\sum_{i=1}^{n} a_{n i} \widetilde{x}_{i} \widetilde{y}_{i} / U_{n}^{2}, \quad \bar{g}_{n}(t)=\sum_{i=1}^{n} W_{n i}(t)\left(y_{i}-x_{i} \bar{\beta}_{n}\right),
$$

where $U_{n}^{2}=\sum_{i=1}^{n} a_{n i} \widetilde{x}_{i}^{2}$, and $a_{n i}=1 / \widehat{f}_{n}\left(u_{i}\right), 1 \leq i \leq n$.

In the sequel, let $C, C_{1}, C_{2}, \ldots$ denote positive constant whose values may vary at each occurrence. Now, we shall give some assumptions:

(C1) (i) For some large natural number $N$, when $n \geq N, C_{1} \leq \frac{S_{n}^{2}}{n} \leq C_{2}$;

(ii) $\max _{1 \leq i \leq n}\left|\widetilde{x}_{i}\right|=O\left(n^{1 / 2}(\log n)^{-1}\right)$.

(C2) (i) $0<m_{0} \leq \min _{1 \leq i \leq n} f\left(u_{i}\right) \leq \max _{1 \leq i \leq n} f\left(u_{i}\right) \leq M_{0}<\infty$;

(ii) $g(\cdot)$ satisfies the first order Lipschitz condition on $I$;

(iii) $\quad f(\cdot)$ and $g(\cdot)$ are continuous functions on $I$.

(C3) $\max _{1 \leq i \leq n} \sum_{j=1}^{n}\left|W_{n j}\left(t_{i}\right)\right|=O(1)$,

$\max _{1 \leq i, j \leq n}\left|W_{n j}\left(t_{i}\right)\right|=O\left(n^{-\frac{1}{2}}(\log n)^{-1}\right)$.

(C4) (i) $\max _{1 \leq i<n}\left|\sum_{j=1}^{n} W_{n j}\left(t_{i}\right)-1\right|=o(1)$;

(ii) $\max _{1 \leq i \leq n} \sum_{j=1}^{n}\left|W_{n j}\left(t_{i}\right)\right| I\left(\left|t_{i}-t_{j}\right|>\delta\right)=o(1), \forall \delta>0$, where $I(E)$ denotes the indicator function of the set $E$.

(C5) (i) $\max _{1 \leq i \leq n}\left|\sum_{j=1}^{n} W_{n j}\left(t_{i}\right)-1\right|=O\left(n^{-\frac{1}{4}}\right)$;

(ii) $\max _{1 \leq i \leq n} \sum_{j=1}^{n}\left|W_{n j}\left(t_{i}\right)\right| I\left(\left|t_{i}-t_{j}\right|>a n^{-\frac{1}{4}}\right)=O\left(n^{-\frac{1}{4}}\right)$ for some $a>0$.

(C6) $\widehat{W}_{n i}(\cdot)(1 \leq i \leq n)$ satisfy (C3) and (C4), in this moment, $u_{i}$ and $\widehat{W}_{n i}(\cdot)$ instead of $t_{i}$ and $W_{n i}(\cdot)$, respectively.

Remark 2.1: From (C1) $(i)$ and (C2) $(i)$, we obtain that, when $n$ is large enough,

$$
C_{3} \leq \frac{T_{n}^{2}}{n} \leq C_{4}, \quad T_{n}^{-2} \sum_{i=1}^{n}\left|a_{i} \widetilde{x}_{i}\right| \leq C, \quad S_{n}^{-2} \sum_{i=1}^{n}\left|\widetilde{x}_{i}\right| \leq C .
$$

\section{Main Results}

When the errors $e_{i}$ are i.i.d., Chen et al. [10] showed the strong consistency for the estimators $\widehat{\beta}_{n}, \widetilde{\beta}_{n}$ and $\bar{\beta}_{n}$ of $\beta$ and the uniform strong consistency for the estimator $\widehat{f}_{n}(\cdot)$ of $f(\cdot)$. The results are summarized below.

Theorem 3.1: ([10]) For the model (1.1), let $\left\{e_{i}, i \geq 1\right\}$ be a sequence of i.i.d. random errors with $E e_{1}=0$ and $E e_{1}^{2}=1$. Suppose that $(C 1),(C 2)(i)($ iiii $),(C 3)$ and $(C 4)$ hold. Then

$$
\begin{gathered}
\widehat{\beta}_{n} \rightarrow \beta \text { a.s. } \\
\widetilde{\beta}_{n} \rightarrow \beta \text { a.s. }
\end{gathered}
$$


Theorem 3.2: ([10]) For the model (1.1), let $\left\{e_{i}, i \geq 1\right\}$ be a sequence of i.i.d. random errors with $E e_{1}=0$ and $E e_{1}^{2}=1$. Suppose that $(C 1),(C 2),(C 3),(C 5)$ and $(C 6)$ hold. If $E\left|e_{1}\right|^{4}<\infty$, then

$$
\begin{gathered}
\widehat{\beta}_{n}-\beta=O\left(n^{-\frac{1}{4}}\right) \text { a.s. } \\
\max _{\leq i \leq n}\left|\widehat{f}_{n}\left(u_{i}\right)-f\left(u_{i}\right)\right| \rightarrow 0 \text { a.s. } \\
\bar{\beta}_{n} \rightarrow \beta \text { a.s. }
\end{gathered}
$$

The main aim of this paper is to investigate whether Theorems 3.1 and 3.2 still hold when the random errors $e_{i}, 1 \leq i \leq n$ are NA random variables.

Definition: A finite family of random variables $\left\{X_{i}, 1 \leq 1 \leq n\right\}$ is said to be negatively associated (NA) if for every pair of disjoint subsets $A$ and $B$ of $\{1,2, \cdots, n\}$,

$$
\operatorname{Cov}\left(f_{1}\left(X_{i}, i \in A\right), f_{2}\left(X_{j}, j \in B\right)\right) \leq 0
$$

whenever $f_{1}$ and $f_{2}$ are coordinatewise increasing and such that the covariance exists. An infinite family of random variables is NA if every finite subfamily is NA.

The notion of negative association was first introduced by Alam and Saxena [1] and studied in detail by Joag-Dev and Proschan [17]. Because of its wide applications in multivariate statistical analysis and systems reliability, the notion of NA have recently received considerable attention. For convergence results, we refer to Joag-Dev and Proschan [17] for fundamental properties, Matula [21] for the three series theorem, Shao and Su [24] for law of the iterated logarithm, Liang [18] for complete convergence, and Liang and Jing [19] for strong laws. Asymptotic properties of estimates related to NA samples have also been studied extensively. Cai and Roussas [3] studied uniformly strong consistency, convergence rates, and asymptotic distribution of Kaplan-Meier estimator of distributed function with random censored failure times, and Cai and Roussas [4] gave Berry-Esseen bounds for smooth estimates of a distribution function. Roussas [23] investigated consistency of the kernel estimate of a probability density function. Amini and Bozorgnia [2] dealt with the consistency and complete convergence of sample quantiles.

However, there have been few asymptotic results for the estimators of parametric and nonparametric component in partial linear model regression under negatively associated error's structure. In the next two theorems, we shall give some results similar to those in Theorems 3.1 and 3.2 under negatively associated error's structure. Furthermore, we wish to consider the uniformly strong consistency for the estimator of $g(\cdot)$ under NA random errors. But first, we list a weaker assumption than (C3):

(C3') $\max _{1 \leq i \leq n} \sum_{j=1}^{n}\left|W_{n j}\left(t_{i}\right)\right|=O(1)$, $\max _{1 \leq i, j \leq n}\left|W_{n j}\left(t_{i}\right)\right|=O\left(n^{-\frac{1}{2}}\right)$.

Our main results are as follows:

Theorem 3.3: For model (1.1), let $\left\{e_{i}, i \geq 1\right\}$ be a sequence of identically distributed zero mean NA random errors. Suppose that $(C 1)(i),(C 2)(i)(i i i),\left(C 3^{\prime}\right)$ and $(C 4)$ hold. Assume that $E e_{1}^{2}<\infty$.

(i) The relations (3.3) and (3.4) remain true.

(ii) If we further assume $\max _{1 \leq j \leq n}\left|\sum_{i=1}^{n} W_{n i}\left(t_{j}\right) x_{i}\right|=O(1)$, then

$$
\begin{aligned}
& \max _{1 \leq i \leq n}\left|\widehat{g}_{n}\left(t_{i}\right)-g\left(t_{i}\right)\right| \rightarrow 0 \text { a.s. } \\
& 1 \leq i \leq n \\
& \max _{1 \leq}\left|\widetilde{g}_{n}\left(t_{i}\right)-g\left(t_{i}\right)\right| \rightarrow 0 \text { a.s. }
\end{aligned}
$$


Theorem 3.4: For model (1.1), let $\left\{e_{i}, i \geq 1\right\}$ be a sequence of zero mean identically distributed NA random errors with $E e_{1}^{2}=1$. Suppose that $(C 1)(i),(C 2),(C 3),(C 5)$ and (C6) hold. Assume that $E e_{1}^{4}<\infty$.

(i) The relations (3.5)-(3.7) are valid.

(ii) If we further assume $\max _{1 \leq j \leq n}\left|\sum_{i=1}^{n} W_{n i}\left(t_{j}\right) x_{i}\right|=O(1)$, then

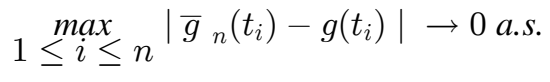

Remark 3.1: Since independent random samples are a special case of NA random samples and (C3') is weaker than (C3), Theorems 3.3-3.4 extend Theorems 3.1-3.2 to NA sample setting and weaken the restriction for the weight function. Moreover, we eliminate the condition (C1) $(i i)$ in Theorems 3.1-3.2.

\section{Proofs of the Main Results}

In this section, $a \ll b$ means $a=O(b)$, $^{+}=\max (0, a), a^{-}=\max (0,-a)$.

Lemma 4.1: Let $\left\{X_{i}, i \geq 1\right\}$ be a sequence of identically distributed NA random variables with $E X_{i}=0$. Assume that $\left\{b_{n i}, 1 \leq i \leq k_{n}, n \geq 1\right\}$ is an array of real numbers, where $k_{n}$ is a sequence of positive integers with $k_{n}=O(n)$.

(1) If for some $p>2, E\left|X_{1}\right|^{p}<\infty$ and $\max _{1 \leq i \leq k_{n}}\left|b_{n i}\right|=O\left(n^{-1 / p}\right)$, $\sum_{i=1}^{k_{n}} b_{n i}^{2}=O\left((\log n)^{-1}\right)$, then $\sum_{i=1}^{k_{n}} b_{n i} X_{i}=O(1)$ a.s.

(2) If $E X_{1}^{2}<\infty$ and $\max _{1 \leq i \leq k_{n}}\left|b_{n i}\right|=O\left(n^{-1 / 2}\right), \sum_{i=1}^{k_{n}} b_{n i}^{2}=O\left(n^{-\delta}\right)$ for any $0<\delta<1$, then $\sum_{i=1}^{k_{n}} b_{n i} X_{i}=o(1)$ a.s.

Lemma 4.2: Let $\left\{\xi_{i}, i \geq 1\right\}$ be a sequence of identically distributed $N A$ random variables with $E \xi_{i}=0$ and $E \xi_{i}^{2}<\infty$. Assume that $\left\{a_{n i}(\cdot), 1 \leq i \leq n, n \geq 1\right\}$ is a weight function array defined on closed interval $I$ of $R$ satisfying $\max _{1 \leq i, j \leq n}\left|a_{n i}\left(u_{j}\right)\right|=O\left(n^{-1 / 2}\right)$, $\max _{1 \leq j \leq n} \sum_{i=1}^{n}\left|a_{n i}\left(u_{j}\right)\right|=O(1)$. Then

$$
\max _{1 \leq j \leq n}\left|\sum_{i=1}^{n} a_{n i}\left(u_{j}\right) \xi_{i}\right|=o(1) \text { a.s., } \max _{1 \leq j \leq n}\left(\sum_{i=1}^{n}\left|a_{n i}\left(u_{j}\right) \xi_{i}\right|\right)=O(1) \text { a.s. }
$$

For proofs of Lemma 4.1 and 4.2, see Liang and Jing [19].

Denote by $\widetilde{\epsilon}_{i}=\epsilon_{i}-\sum_{j=1}^{n} W_{n j}\left(t_{i}\right) \epsilon_{j}, \widetilde{g}_{i}=g\left(t_{i}\right)-\sum_{j=1}^{n} W_{n j}\left(t_{i}\right) g\left(t_{j}\right), \epsilon_{i}=\sigma_{i} e_{i}$, then it is easy to see

$$
\begin{gathered}
\widehat{\beta}_{n}-\beta=S_{n}^{-2}\left[\sum_{i=1}^{n} \widetilde{x}_{i} \widetilde{\epsilon}_{i}+\sum_{i=1}^{n} \widetilde{x}_{i} \widetilde{g}_{i}\right], \\
\widetilde{\beta}_{n}-\beta=T_{n}^{-2}\left[\sum_{i=1}^{n} a_{i} \widetilde{x}_{i} \widetilde{\epsilon}_{i}+\sum_{i=1}^{n} a_{i} \widetilde{x}_{i} \widetilde{g}_{i}\right], \\
\bar{\beta}_{n}-\beta=U_{n}^{-2}\left[\sum_{i=1}^{n} a_{n i} \widetilde{x}_{i} \widetilde{\epsilon}_{i}+\sum_{i=1}^{n} a_{n i} \widetilde{x}_{i} \widetilde{g}_{i}\right],
\end{gathered}
$$


Proof of Theorem 3.3: (i) We prove only (3.4) as the proof of (3.3) is analogous. From (4.12), we get

$$
\widetilde{\beta}_{n}-\beta=T_{n}^{-2}\left[\sum_{i=1}^{n} a_{i} \widetilde{x}_{i} \epsilon_{i}-\sum_{i=1}^{n} a_{i} \widetilde{x}_{i}\left(\sum_{j=1}^{n} W_{n j}\left(t_{i}\right) \epsilon_{j}\right)+\sum_{i=1}^{n} a_{i} \widetilde{x}_{i} \widetilde{g}_{i}\right]=: A_{1 n}+A_{2 n}+A_{3 n}
$$

Note that $A_{1 n}=\sum_{i=1}^{n}\left(T_{n}^{-2} \cdot a_{i} \widetilde{x}_{i} \sigma_{i}\right) e_{i}=: \sum_{i=1}^{n} b_{n i} e_{i}$. By conditions $(\mathrm{C} 1)(i)$,

(C2) $(i)$ and (2.2) we know $\max _{1 \leq i \leq n}\left|b_{n i}\right|=O\left(n^{-1 / 2}\right), \sum_{i=1}^{n} b_{n i}^{2}=O\left(n^{-1}\right)$. Hence, by Lemma 4.1(2), we et $A_{1 n} \rightarrow 0$ a.s.

Obviously, $A_{2 n}=\sum_{j=1}^{n}\left(\sum_{i=1}^{n} T_{n}^{-2} a_{i} \widetilde{x}_{i} W_{n j}\left(t_{i}\right) \sigma_{j}\right) e_{j}=: \sum_{j=1}^{n} C_{n j} e_{j}$. By conditions (C2) $(i), \quad\left(\mathrm{C}^{\prime}\right)$ and (2.2), we get $\max _{1 \leq j \leq n}\left|C_{n j}\right|-O\left(n^{-1 / 2}\right), \quad \sum_{j=1}^{n}$ $C_{n j}^{2}=O\left(n^{-1 / 2}\right)$. Hence, by Lemma 4.1(2), we get $A_{2 n} \rightarrow 0$ a.s.

As to $A_{3 n}$, we note that $\left|A_{3 n}\right| \leq\left(\max _{1 \leq j \leq n}\left|\widetilde{g}_{i}\right|\right)\left(T_{n}^{-2} \sum_{i=1}^{n}\left|a_{i} \widetilde{x}_{i}\right|\right)$ and

$$
\begin{aligned}
& \max _{1 \leq i \leq n}\left|\widetilde{g}_{i}\right| \leq \max _{1 \leq i \leq n}\left|g\left(t_{i}\right)\right|\left|\sum_{j=1}^{n} W_{n j}\left(t_{i}\right)-1\right| \\
& +\max _{1 \leq i \leq n_{j=1}}\left|W_{n j}\left(t_{i}\right)\right|\left|g\left(t_{i}\right)-g\left(t_{j}\right)\right| I\left(t_{i}-t_{j} \mid>\delta\right) \\
& +\max _{1 \leq i \leq n_{j=1}^{n}\left|W_{n j}\left(t_{i}\right)\right|\left|g\left(t_{i}\right)-g\left(t_{j}\right)\right| I\left(t_{i}-t_{j} \mid \leq \delta\right) .}
\end{aligned}
$$

So, by the conditions (C2) $(i i i),\left(\mathrm{C}^{\prime}\right)$ and (C4), we get

$$
\max _{1 \leq i \leq n}\left|\widetilde{g}_{i}\right| \rightarrow 0
$$

which, together with (2.2), implies $A_{3 n} \rightarrow 0$. Therefore, Theorem 3.3 $(i)$ is proved.

(ii) Again, we only prove (3.8) as the proof of (3.9) is similar. It is easy to see

$$
\begin{aligned}
& \max _{1 \leq i \leq n}\left|\widehat{g}_{n}\left(t_{i}\right)-g\left(t_{i}\right)\right| \\
& \qquad \max _{1 \leq i \leq n}\left\{\left|\beta-\widehat{\beta}_{n}\right|\left|\sum_{j=1}^{n} W_{n j}\left(t_{i}\right) x_{i}\right|+\left|\widetilde{g}_{i}\right|+\left|\sum_{j=1}^{n} W_{n j}\left(t_{i}\right) \sigma_{j} e_{j}\right|\right\} \\
& =: B_{1 n}+B_{2 n}+B_{3 n} .
\end{aligned}
$$

By (3.3) and assumption, we get $B_{1 n} \rightarrow 0$ a.s. From (C2) $(i)$ and (C3'), using Lemma 4.2, we have $B_{3 n} \rightarrow 0$ a.s. Therefore, (3.8) is proved from (4.15).

Proof of Theorem 3.4: (i) We first prove (3.5). From (4.11), we have

$$
\widehat{\beta}_{n}-\beta=S_{n}^{-2}\left[\sum_{i=1}^{n} \widetilde{x}_{i} \widetilde{g}_{i}+\sum_{i=1}^{n} \widetilde{x}_{i} \epsilon_{i}-\sum_{i=1}^{n} \widetilde{x}_{i}\left(\sum_{j=1}^{n} W_{n j}\left(t_{i}\right) \epsilon_{j}\right)\right]=: H_{1 n}+H_{2 n}+H_{3 n} .
$$


Note that $\left|H_{1 n}\right| \leq\left(\max _{1 \leq j \leq n}\left|\widetilde{g}_{i}\right|\right)\left(\sum_{i=1}^{n}\left|\widetilde{x}_{i}\right| / T_{n}^{2}\right) 1$. Now similarly to the proof in (4.14), we get

$$
\max _{1 \leq j \leq n}\left|\widetilde{g}_{i}\right|=O\left(n^{-1 / 4}\right)
$$

Hence, (4.16) and (2.2) imply $H_{1 n}=O\left(n^{-1 / 4}\right)$. Note that

$$
\begin{gathered}
H_{2 n}=\sum_{i=1}^{n}\left(S_{n}^{-2} \cdot \widetilde{x}_{i} \sigma_{i}\right) e_{i}=: \sum_{i=1}^{n} D_{n i} e_{i}, \\
H_{3 n}=\sum_{j=1}^{n}\left(S_{n}^{-2} \sum_{i=1}^{n} \widetilde{x}_{i} W_{n j}\left(t_{i}\right) \sigma_{j}\right) e_{j}=: \sum_{j=1}^{n} E_{n j} e_{j} .
\end{gathered}
$$

From conditions (C1)(i), (C2)(i), (C3) and (2.2), we have $\max _{1 \leq i \leq n}\left|D_{n i}\right|=O\left(n^{-1 / 2}\right)$, $\sum_{i=1}^{n} D_{n i}^{2}=O\left(n^{-1}\right), \max _{1 \leq j \leq n}\left|E_{n j}\right|=O\left(n^{-1 / 2}(\log n)^{-1}\right)$, $\sum_{j=1}^{n} E_{n j}^{2}=O\left(n^{-1 / 2}(\log n)^{-1}\right)$. Hence by Lemma 4.1 (1), we get $H_{2 n}=o\left(n^{-1 / 4}\right)$ and $H_{3 n}=O\left(n^{-1 / 4}\right)$, respectively.

Next we prove (3.6). Note that $\widehat{f}_{n}(u)=\sum_{i=1}^{n} \widehat{W}_{n i}(u)\left[\widetilde{x}_{i}\left(\beta-\widehat{\beta}_{n}\right)+\widetilde{g}_{i}+\widetilde{\epsilon}_{i}\right]^{2}$. Therefore,

$$
\begin{aligned}
& \max _{1 \leq j \leq n}\left|\widehat{f}_{n}\left(u_{j}\right)-f\left(u_{j}\right)\right| \\
& \leq_{1 \leq j \leq n}\left|\sum_{i=1}^{n} \widehat{W}_{n i}\left(u_{j}\right) \epsilon_{i}^{2}-f\left(u_{j}\right)\right|+\underset{1 \leq j \leq n}{2} \max _{i=1}\left|\sum_{i=1}^{n} \widehat{W}_{n i}\left(u_{j}\right) \epsilon_{i}\left(\sum_{j=1}^{n} W_{n j}\left(t_{i}\right) \epsilon_{j}\right)\right| \\
& +\max _{1 \leq j \leq n}\left|\sum_{i=1}^{n} \widehat{W}_{n i}\left(u_{j}\right)\left(\sum_{j=1}^{n} W_{n j}\left(t_{i}\right) \epsilon_{j}\right)^{2}+\underset{1 \leq j \leq n}{2} \max _{i=1}\right| \sum_{i=1}^{n} \widehat{W}_{n i}\left(u_{j}\right) \widetilde{g}_{i} \widetilde{\epsilon}_{i} \mid \\
& +\underset{1}{2} \max _{j \leq n}\left|\sum_{i=1}^{n} \widehat{W}_{n i}\left(u_{j}\right) \widetilde{x}_{i} \widetilde{\epsilon}_{i}\right|\left|\beta-\widehat{\beta}_{n}\right|+\left(\beta-\widehat{\beta}_{n}\right)_{1}^{2} \cdot \max _{\leq j}\left|\sum_{i=1}^{n} \widehat{W}_{n i}\left(u_{j}\right) \widetilde{x}_{i}^{2}\right| \\
& +2\left|\beta-\widehat{\beta}_{n}\right|_{1} \cdot \max _{j \leq n}\left|\sum_{i=1}^{n} \widehat{W}_{n i}\left(u_{j}\right) \widetilde{x}_{i} \widetilde{g}_{i}\right|+\max _{1 \leq j \leq n}\left|\sum_{i=1}^{n} \widehat{W}_{n i}\left(u_{j}\right) \widetilde{g}_{i}^{2}\right| \\
& =: L_{1 n}+L_{2 n}+L_{3 n}+L_{4 n}+L_{5 n}+L_{6 n}+L_{7 n}+L_{8 n} .
\end{aligned}
$$

For $L_{1 n}$ we have

$$
\begin{gathered}
\left|L_{1 n}\right| \leq \max _{1 \leq j \leq n}\left|\sum_{i=1}^{n} \widehat{W}_{n i}\left(u_{j}\right) f\left(u_{i}\right)\left(e_{i}^{2}-1\right)\right| \\
+\max _{1 \leq j \leq n}\left|\sum_{i=1}^{n} \widehat{W}_{n i}\left(u_{j}\right) f\left(u_{i}\right)-f\left(u_{j}\right)\right|=: L_{11 n}+L_{12 n} .
\end{gathered}
$$


Noticing that $E e_{1}^{2}=1$, so, $e_{i}^{2}-1=\left[\left(e_{i}^{+}\right)^{2}-E\left(e_{i}^{+}\right)^{2}\right]+\left[\left(e_{i}^{-}\right)^{2}-E\left(e_{i}^{-}\right)^{2}\right]=: \eta_{i}+\xi_{i}$ and

$$
L_{11 n} \leq \max _{1}\left|\sum_{i=1}^{n} \widehat{W}_{n i}\left(u_{j}\right) f\left(u_{i}\right) \eta_{i}\right|+\max _{1 \leq j \leq n}\left|\sum_{i=1}^{n} \widehat{W}_{n i}\left(u_{j}\right) f\left(u_{i}\right) \xi_{i}\right| .
$$

Since $\left\{\eta_{i}, i \geq 1\right\}$ and $\left\{\xi_{i}, i \geq 1\right\}$ are NA random variables with $E \eta_{1}=0, E \eta_{1}^{2} \ll E e_{1}^{4}<\infty$, $E \xi_{1}=0, E \xi_{1}^{2}<\infty$ and

$$
\max _{1 \leq j \leq n}\left|\widehat{W}_{n i}\left(u_{j}\right) f\left(u_{i}\right)\right|=O\left(n^{-1 / 2}(\log n)^{-1}\right), \max _{1} \sum_{i=1}^{n}\left|\widehat{W}_{n i}\left(u_{j}\right) f\left(u_{i}\right)\right|=O(1) .
$$

By Lemma 4.2, we get $L_{11 n} \rightarrow 0$ a.s. Applying the methods of proof in (4.14), by (C2)(iii) and (C6), we can get $L_{12 n} \rightarrow 0$ a.s. Therefore $L_{1 n} \rightarrow 0$ a.s.

Note that $\left|L_{2 n}\right| \leq 2\left(\max _{1 \leq i \leq n}\left|\sum_{j=1}^{n} W_{n j}\left(t_{i}\right) \sigma_{j} e_{j}\right|\right) \quad\left(\max _{1 \leq j \leq n} \sum_{i=1}^{n}\right.$ $\left.\left|\widehat{W}_{n i}\left(u_{j}\right) \sigma_{i} e_{i}\right|\right)$. Hence, by Lemma 4.2 , we get $L_{2 n} \rightarrow 0$ a.s.

From the assumptions of the theorem and from (4.16), (2.2), (3.5) and Lemma 4.2, we have

$$
\begin{aligned}
& \left|L_{3 n}\right| \leq\left(\max _{1 \leq i \leq n}\left(\sum_{j=1}^{n} W_{n j}\left(t_{i}\right) \sigma_{j} e_{j}\right)^{2}\right)\left(\max _{1 \leq j \leq n_{i=1}^{n}}\left|\widehat{W}_{n i}\left(u_{j}\right)\right|\right) \rightarrow 0 \text { a.s. } \\
& \left.\left|L_{4 n}\right| \leq 2\left\{\left(\max _{1 \leq i \leq n}\left|\widetilde{g}_{i}\right|\right)\right\} \max _{1 \leq j \leq n_{i=1}}\left|\widehat{W}_{n i}\left(u_{j}\right) \epsilon_{i}\right|\right) \\
& \left.\left.+\left(\max _{1 \leq i \leq n}\left|\widetilde{g}_{i}\right|\right)\left\{\max _{\leq i \leq n} \mid \sum_{k=1}^{n} W_{n k}\left(t_{i}\right) \epsilon_{k}\right) \mid\right)\left(\max _{1 \leq j \leq n_{i=1}} \sum^{n}\left|\widehat{W}_{n i}\left(u_{j}\right)\right|\right)\right\} \rightarrow 0 \text { a.s. } \\
& \left|L_{6 n}\right| \leq\left(\widehat{\beta}_{n}-\beta\right)^{2}\left(\max _{1 \leq i, j \leq n}\left|\widehat{W}_{n i}\left(u_{j}\right)\right|\right)\left(\sum_{i=1}^{n} \widetilde{x}_{i}^{2}\right)=o(1) \text { a.s. } \\
& \left.\left|L_{7 n}\right| \leq 2\left|\beta-\widehat{\beta}_{n}\right| \max _{1 \leq j \leq n}\left|\widetilde{g}_{i}\right|\right\}_{1}\left(\max _{i, j \leq n}\left|\widehat{W}_{n i}\left(u_{j}\right)\right|\right)\left(\sum_{i=1}^{n}\left|\widetilde{x}_{i}\right|\right)=o(1) \text { a.s. } \\
& \left.\left.\left|L_{8 n}\right| \leq\left(\max _{1 \leq j \leq n} \widetilde{g}_{i}^{2}\right)\right\} \max _{\leq j \leq n_{i=1}}\left|\widehat{W}_{n i}\left(u_{j}\right)\right|\right) \rightarrow 0 \\
& \left|L_{5 n}\right| \leq 2\left|\beta-\widehat{\beta}_{n}\right|_{1 \leq j \leq n} \sqrt{\left(\sum_{i=1}^{n} \widehat{W}_{n i}^{2}\left(u_{j}\right) \widetilde{e}_{i}^{2}\right)\left(\sum_{i=1}^{n} \widetilde{x}_{i}^{2}\right)} .
\end{aligned}
$$

To prove $L_{5 n} \rightarrow 0$ a.s., it suffices to show that

$$
\max _{j \leq n}\left(\sum_{i=1}^{n} \widehat{W}_{n i}^{2}\left(u_{j}\right) \widetilde{e}_{i}^{2}\right)=o\left(n^{-1 / 2}\right) \text { a.s. }
$$


Note that

$$
\begin{aligned}
& \max _{1 \leq j \leq n}\left(\sum_{i=1}^{n} \widehat{W}_{n i}^{2}\left(u_{j}\right) \widetilde{\epsilon}_{j}^{2}\right) \\
& \leq_{1 \leq j \leq n}\left(\sum_{i=1}^{n} \widehat{W}_{n i}^{2}\left(u_{j}\right) \epsilon_{i}^{2}\right)+\underset{1}{2} \max _{j} \leq n\left|\sum_{i=1}^{n} \widehat{W}_{n i}^{2}\left(u_{j}\right) \epsilon_{i}\left(\sum_{k=1}^{n} W_{n k}\left(t_{i}\right) \epsilon_{k}\right)\right| \\
& +\left\{\max _{\leq j \leq n_{i=1}} \sum_{W_{n i}}^{2}\left(u_{j}\right)\right)\left(\sum_{k=1}^{n} W_{n k}\left(t_{i}\right) \epsilon_{k}\right)^{2} \\
& =: L_{51 n}+L_{52 n}+L_{53 n} .
\end{aligned}
$$

By Lemma 4.2 and condition (C6), we get

$$
\begin{aligned}
& \left|L_{52 n}\right| \leq 2\left\{\max _{1 \leq i \leq n}\left|\sum_{k=1}^{n} W_{n k}\left(t_{i}\right) \epsilon_{k}\right|\right)\left(\max _{1} \sum_{j=1}^{n}\left|\widehat{W}_{n i}\left(u_{j}\right) \epsilon_{i}\right|\right)=o\left(n^{-\frac{1}{2}}\right) \text { a.s. } \\
& \left.\left|L_{53 n}\right| \leq \max _{1 \leq i \leq n}\left|\sum_{k=1}^{n} W_{n k}\left(t_{i}\right) \epsilon_{k}\right| \underset{1}{2}\right)\left(\max _{1}\left|\widehat{W}_{n i}\left(u_{j}\right)\right|\right) \\
& \times\left\{\max _{1 \leq j \leq n_{i=1}}\left|\widehat{W}_{n i}\left(u_{j}\right)\right|\right)=o\left(n^{-\frac{1}{2}}\right) \text { a.s. }
\end{aligned}
$$

Note that

$$
\begin{gathered}
\left|L_{51 n}\right| \leq \max _{1 \leq j \leq n}\left|\sum_{i=1}^{n} \widehat{W}_{n i}^{2}\left(u_{j}\right) \sigma_{i}^{2}\left(\left(e_{i}^{+}\right)^{2}-E\left(e_{i}^{+}\right)^{2}\right)\right| \\
+\max _{1 \leq j \leq n}\left|\sum_{i=1}^{n} \widehat{W}_{n i}^{2}\left(u_{j}\right) \sigma_{i}^{2}\left(\left(e_{i}^{-}\right)^{2}-E\left(e_{i}^{-}\right)^{2}\right)\right|+\max _{1 \leq j \leq n}\left|\sum_{i=1}^{n} \widehat{W}_{n i}^{2}\left(u_{j}\right) \sigma_{i}^{2} E e_{i}^{2}\right| .
\end{gathered}
$$

Since $\left\{\left(e_{i}^{+}\right)^{2}-E\left(e_{i}^{+}\right)^{2}, i \geq 1\right\}$ and $\left\{\left(e_{i}^{-}\right)^{2}-E\left(e_{i}^{-}\right)^{2}, i \geq 1\right\}$ are zero mean NA random variables, it follows by Lemma 4.2, (C6) and

$$
\begin{aligned}
\max _{1 \leq j \leq n}\left|\sum_{i=1}^{n} \widehat{W}_{n i}^{2}\left(u_{j}\right) \sigma_{i}^{2} E e_{i}^{2}\right| & \\
& \left.\leq\left(\max _{1 \leq j \leq n}\left|\widehat{W}_{n i}\left(u_{j}\right) \sigma_{i}^{2} E e_{i}^{2}\right|\right)_{1 \leq j \leq n_{i=1}}\left|\widehat{W}_{n i}\left(u_{j}\right)\right|\right)=o\left(n^{-\frac{1}{2}}\right)
\end{aligned}
$$

that $L_{51 n}=o\left(n^{-1 / 2}\right)$ a.s. Thus, (4.17) is verified.

We now prove (3.7). From (3.6) and condition (C2)( $i$ ), we know that, when $n$ is large enough, 


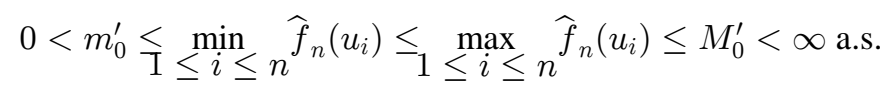

and $C_{6} \leq \frac{U_{n}^{2}}{n} \leq C_{7}, U_{n}^{-2} \sum_{i=1}^{n}\left|a_{n i} \widetilde{x}_{i}\right| \leq C$. By (4.13), we get

$$
\left|\bar{\beta}_{n}-\beta\right| \leq \frac{n}{U_{n}^{2}}\left|\frac{1}{n} \sum_{i=1}^{n} a_{n i} \widetilde{x}_{i} \widetilde{\epsilon}_{i}\right|+\frac{1}{U_{n}^{2}}\left|\sum_{i=1}^{n} a_{n i} \widetilde{x}_{i} \widetilde{g}_{i}\right|=: \frac{n}{U_{n}^{2}} Q_{1 n}+Q_{2 n} .
$$

By (4.15) and (4.19) we have

$$
\begin{gathered}
\left.Q_{2 n} \leq \max _{1 \leq i \leq n}\left|\widetilde{g}_{n}\right|\right)\left(U_{n}^{-2} \sum_{i=1}^{n}\left|a_{n i} \widetilde{x}_{i}\right|\right) \rightarrow 0 \\
Q_{1 n} \leq\left|\frac{1}{n} \sum_{i=1}^{n}\left({ }_{n i}-a_{i}\right) \widetilde{x}_{i} \widetilde{\epsilon}_{i}\right|+\left|\frac{1}{n} \sum_{i=1}^{n} a_{i} \widetilde{x}_{i} \widetilde{\epsilon}_{i}\right| \\
\leq\left|\frac{1}{n} \sum_{i=1}^{n}\left(a_{n i}-a_{i}\right) \widetilde{x}_{i} \epsilon_{i}\right|+\left|\frac{1}{n} \sum_{i=1}^{n}\left(a_{n i}-a_{i}\right) \widetilde{x}_{i}\left(\sum_{j=1}^{n} W_{n j}\left(t_{i}\right) \epsilon_{j}\right)\right| \\
+\left|\frac{1}{n} \sum_{i=1}^{n} a_{i} \widetilde{x}_{i} \widetilde{\epsilon}_{i}\right|=: Q_{11 n}+Q_{12 n}+Q_{13 n} .
\end{gathered}
$$

By Lemma 4.2, we know that

$$
\begin{gathered}
\frac{1}{n} \sum_{i=1}^{n} e_{i}^{2}=\frac{1}{n} \sum_{i=1}^{n}\left(\left(e_{i}^{+}\right)^{2}-E\left(e_{i}^{+}\right)^{2}\right)+\frac{1}{n} \sum_{i=1}^{n}\left(\left(e_{i}^{-}\right)^{2}-E\left(e_{i}^{-}\right)^{2}\right)+\frac{1}{n} \sum_{i=1}^{n} E e_{i}^{2} \\
\rightarrow E e_{1}^{2} \text { a.s. }
\end{gathered}
$$

Hence, from (3.6), (4.18)-(2.10) and conditions (C1) $(i)$, (C2)(i), using Lemma 4.2, we have

$$
\begin{aligned}
& Q_{11 n} \leq\left\{\max _{\leq i \leq n} \frac{\left|f\left(u_{i}\right)-\widehat{f}_{n}\left(u_{i}\right)\right|}{f^{1 / 2}\left(u_{i}\right) \hat{f}_{n}\left(u_{i}\right)}\right) \sqrt{\left(\frac{1}{n} \sum_{i=1}^{n} \widetilde{x}_{i}^{2}\right)\left(\frac{1}{n} \sum_{i=1}^{n} e_{i}^{2}\right)} \rightarrow 0 \text { a.s. } \\
& \left.Q_{12 n} \leq\left\{\max _{1 \leq i \leq n}\left(\sum_{j=1}^{n} W_{n j}\left(t_{i}\right) \epsilon_{j} \mid\right)\right\} \max _{\leq i \leq n}\left|a_{n i}-a_{i}\right|\right)\left(\frac{1}{n} \sum_{i=1}^{n}\left|\widetilde{x}_{i}\right|\right) \rightarrow \text { a.s. }
\end{aligned}
$$

Note that $Q_{13 n}=\frac{T_{n}^{2}}{n}\left(\left|A_{1 n}+A_{2 n}\right|\right)$, and hence $Q_{12 n} \rightarrow 0$ a.s.

(ii) The proof of (3.10) is similar to that of (3.8) in Theorem 3.3 and hence omitted here.

\section{Acknowledgements}

This research was partially supported by the National Natural Science Foundation of China (No. 10171079) and Hong Kong RGC CERG No. HKUST6148/01P. 


\section{References}

[1] Alam, K. and Saxena, K.M.L., Positive dependence in multivariate distributions, Commun. Statist. Theor. Meth. A10 (1981), 1183-1196.

[2] Amini, M. and Bozorgnia, A., Negatively dependent bounded random variable probability inequalities and the strong law of large numbers, J. Appl. Math. and Stoch. Anal. 13:3 (2000), 261-267.

[3] Cai, Z.W. and Roussas, G.G., Kaplan-Meier estimator under association, J. Multiv. Anal. 76 (1998), 318-348.

[4] Cai, Z.W. and Roussas, G.G., Berry-Esseen bounds for smooth estimator of distribution function under association, Nonpar. Statist. 11 (1999), 79-106.

[5] Carroll, R.J., Adapting for heteroscedasticity in linear models, Ann. Statist. 10:4 (1982), 1224-1232.

[6] Carroll, R.J. and Hardle, W., Second order effects in semiparametric weighted least squares regression, Statistics 2 (1989), 179-186.

[7] Chen, H., Convergent rates for parametric components in a partly linear model, Ann. Statist. 16 (1988), 136-146.

[8] Chen, H. and Shiau, H.J., A two-stage spline smoothing method for partially linear model, J. Statist. Plan. Infer. 27 (1991), 187-201.

[9] Chen, H. and Shiau, J.H., Data-driven efficient estimators for a partially linear model, Ann. Statist. 22 (1994), 211-237.

[10] Chen, M.H., Ren, Z., and Hu, S.H., Strong consistency of a class of estimators in a partial linear model, Acta. Math. Sinica 41:2 (1998), 429-439.

[11] Engle, R., Granger, C., Rice, J., and Weiss, A., Nonparametric estimates of the relation between weather and electricity sales, J. Amer. Statist. Assoc. 81 (1986), 310-320.

[12] Gao, J.T., Chen, X.R., and Zhao, L.C., Asymptotic normality of a class of estimators in partial linear models, Acta. Math. Sinica 37:2 (1994), 256-268.

[13] Hamilton, S.A. and Truong, Y.K., Local linear estimation in partly linear models, J. of Multiv. Anal. 60 (1997), 1-19.

[14] Heckman, N., Spline smoothing in partly linear models, J.R. Statist. Soc. B48 (1986), 244-248.

[15] Hong, S.Y. and Cheng, P., Bootstrap approximation for semiparametric regression models, Sci. in China Ser A 23 (1993), 239-251.

[16] Hong, S.Y. and Cheng, P., The convergence rate of estimation for parameter in a semiparametric model, Chin. J. of Appl. Prob. and Statist. 10 (1994), 62-71.

[17] Joag-Dev, K. and Proschan, F., Negative association of random variables with applications, Ann. Statist. 11 (1983), 286-295.

[18] Liang, H.Y., Complete convergence for weighted sums of negatively associated random variables, Statist. \& Prob. Letters 48 (2000), 317-325.

[19] Liang, H.Y. and Jing, B.Y., Strong laws for weighted sums of NA variables, (2002), manuscript.

[20] Mammen, E. and Van de Geer, Penalized quasi-likelihood estimation in partial linear models, Ann. Statist. 25 (1997), 1014-1035.

[21] Matula, P., A note on the almost sure convergence of sums of negatively dependences random variables, Statist. \& Prob. Letters 15 (1992), 209-212.

[22] Robinson, P.M., Asymptotically efficiency estimation in the presence of heteroscedasticity of unknown form, Econometrika 55 (1987), 875-891.

[23] Roussas, G.G., An Esseen-type inequality for probability density functions with an application, Statist. \& Probab. Letters 51 (2001), 379-408.

[24] Shao, Q.M. and Su, C., The law of the iterated logarithm for negatively associated random variables, Stoch. Process Appl. 83 (1999), 139-148. 
[25] Speckman, P., Kernel smoothing in partial linear models, J.R. Statist. Soc. B 50 (1988), 413-436. 


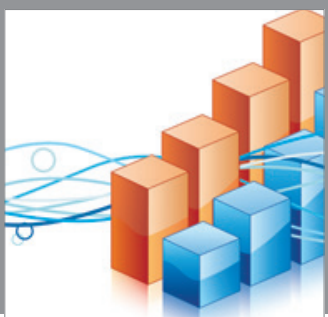

Advances in

Operations Research

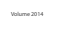

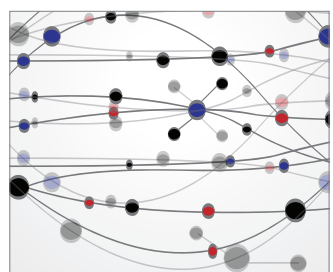

\section{The Scientific} World Journal
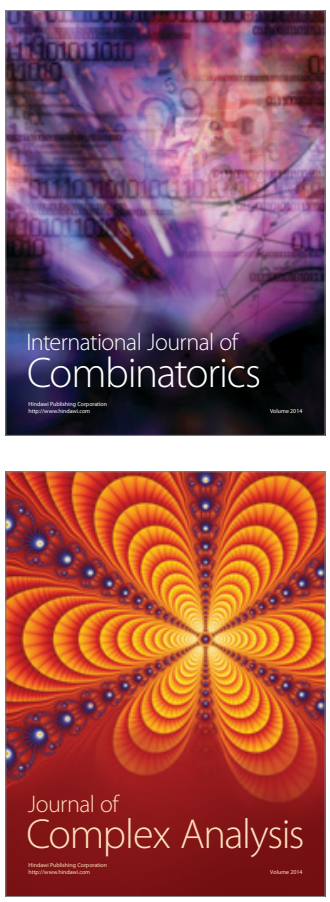

International Journal of

Mathematics and

Mathematical

Sciences
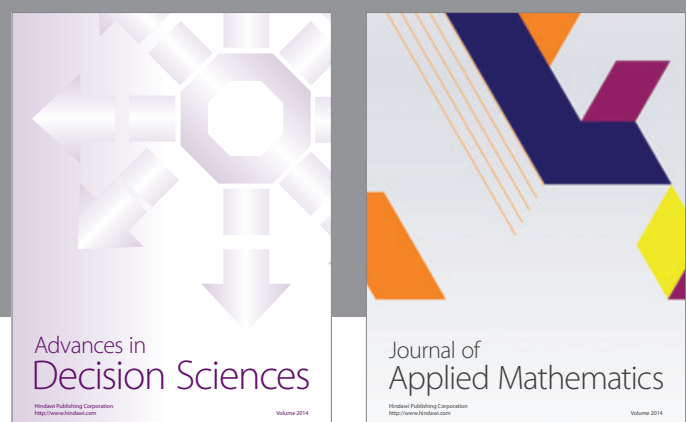

Journal of

Applied Mathematics
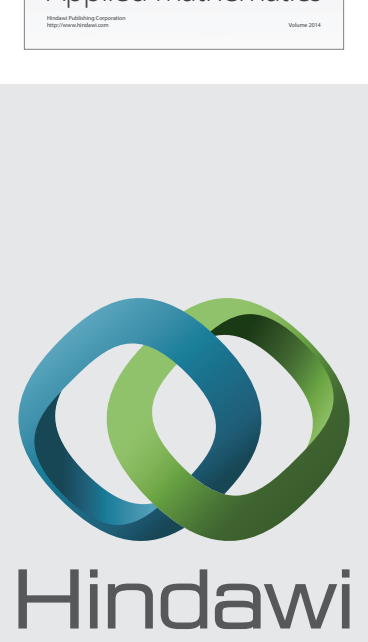

Submit your manuscripts at http://www.hindawi.com
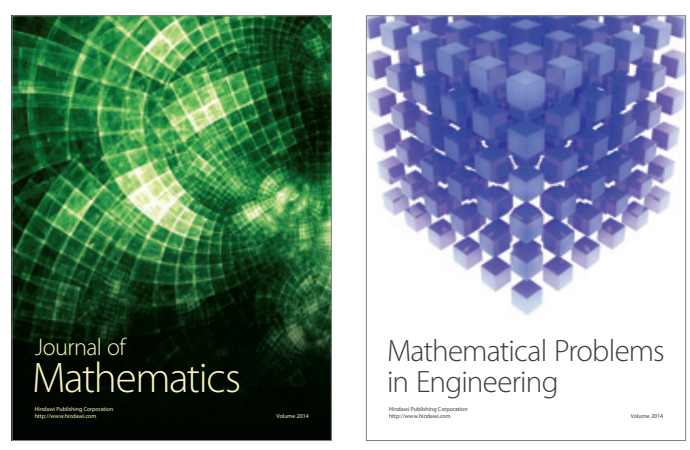

Mathematical Problems in Engineering
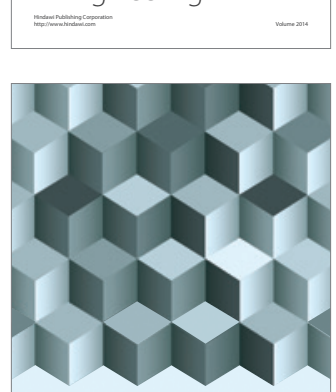

Journal of

Function Spaces
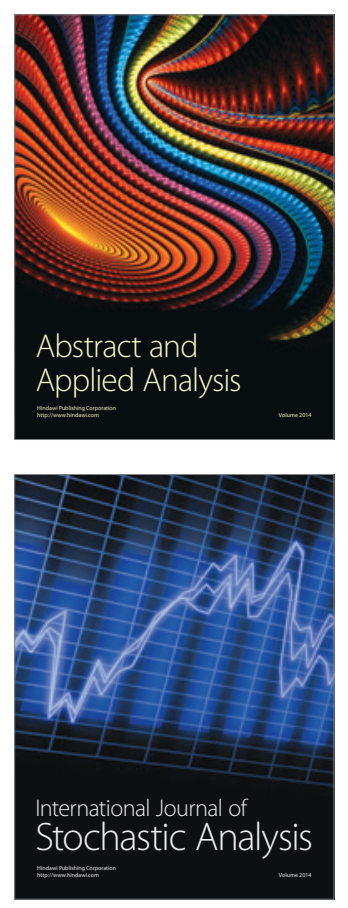

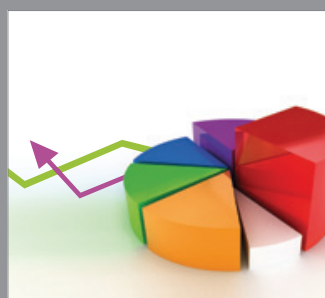

ournal of

Probability and Statistics

Promensencen
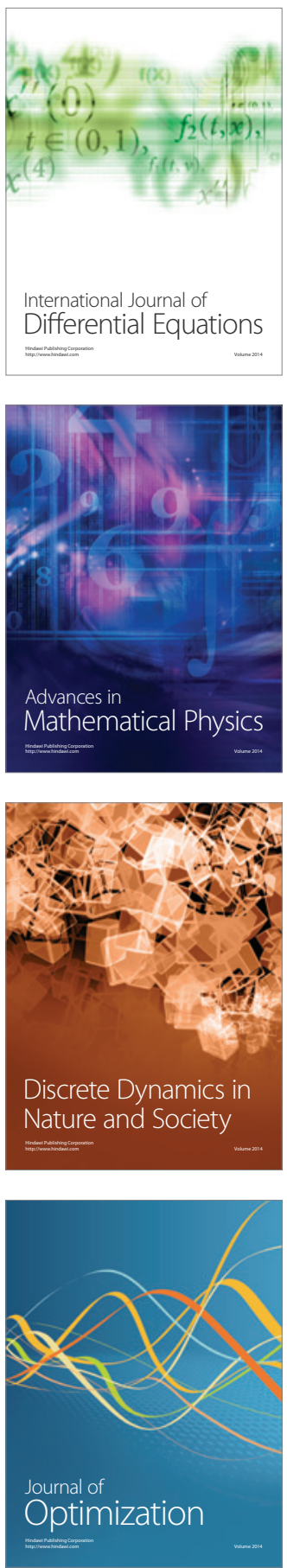